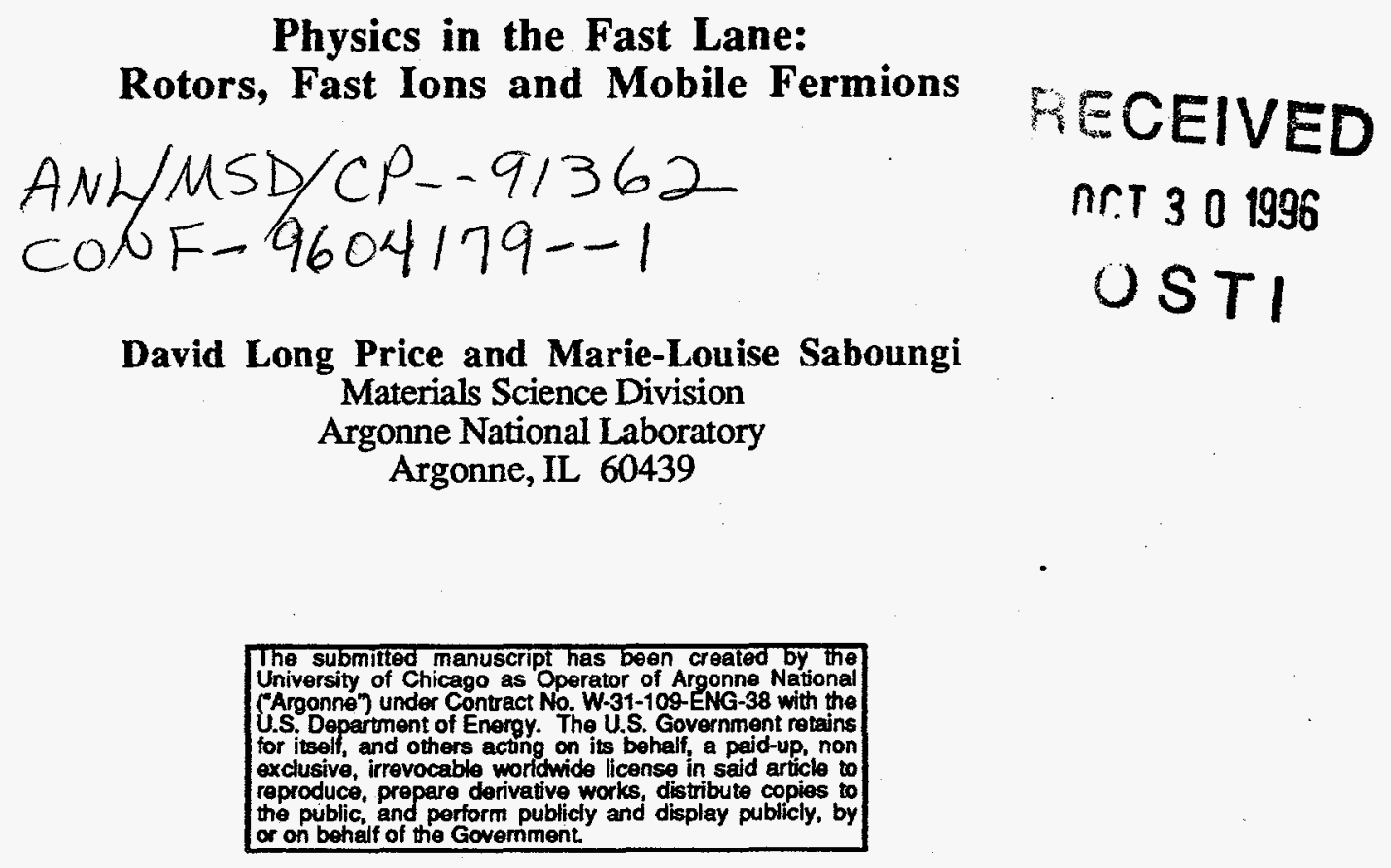

September, 1996

To be published in Journal of Physics: Condensed Matter

$/ \mathrm{sm}$

Distribution:

1-2. M. J. Masek

3. B. D. Dunlap

4. G. W. Crabtree

5. J. D. Jorgensen

6. B. S. Brown $/ 330$

7. Editorial Office

8. Authors

Invited Talk at Symposium on the Physics and Chemistry of Conducting Liquids, Bristol, UK, April 18, 1996

This work is supported by the Division of Materials Sciences, Office of Basic Energy Sciences of DOE, under contract No. W-31-109-ENG-38. 


\section{DISCLAIMER}

Portions of this document may be illegible in electronic image products. Images are produced from the best available original document. 


\section{DISCLAIMER}

This report was prepared as an account of work sponsored by an agency of the United States Government. Neither the United States Government nor any agency thereof, nor any of their employees, makes any warranty, express or implied, or assumes any legal liability or responsibility for the accuracy, completeness, or usefulness of any information, apparatus, product, or process disclosed, or represents that its use would not infringe privately owned rights. Reference herein to any specific commercial product, process, or service by trade name, trademark, manufacturer, or otherwise does not necessarily constitute or imply its endorsement, recommendation, or favoring by the United States Government or any agency thereof. The views and opinions of authors expressed herein do not necessarily state or reflect those of the United States Government or any agency thereof. 


\title{
Physics in the Fast Lane: \\ Rotors, Fast Ions and Mobile Fermions
}

\author{
David Long Price and Marie-Louise Saboungi \\ Materials Science Division \\ Argonne National Laboratory \\ Argonne, IL 60439
}

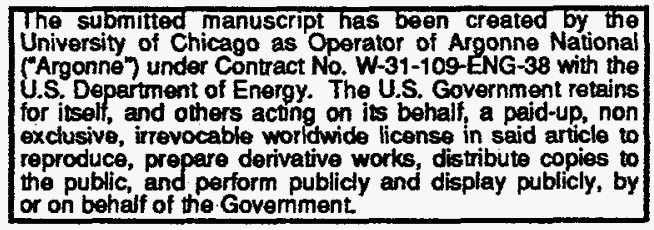

September, 1996

To be published in Journal of Physics: Condensed Matter

$/ \mathrm{sm}$

Invited Talk at Symposium on the Physics and Chemistry of Conducting Liquids, Bristol, UK, April 18, 1996

This work is supported by the Division of Materials Sciences, Office of Basic Energy Sciences of DOE, under contract No. W-31-109-ENG-38. 


\title{
Physics in the Fast Lane: Rotors, Fast Ions and Mobile Fermions
}

\author{
David Long Price and Marie-Louise Saboungi \\ Materials Science Division \\ Argonne National Laboratory \\ Argonne, IL 60439, USA
}

September 1996

Invited Talk at Symposium on the Physics and Chemistry of Conducting Liquids, Bristol, UK, April 18, 1996

To be published in Journal of Physics: Condensed Matter

\begin{abstract}
Dynamic disorder in the high-temperature solid phases of the Zintl compounds $\mathrm{CsPb}$ and $\mathrm{NaSn}$ is characterized by fast orientational motions of the polyanions and coupled motions of the cations Melting is characterized by slow translational motions of the centers of mass of the polyanions. The dynamic behaviour of the ions is associated with dramatic increases in electrical conductivity characteristic of the behavior expected of a mixed conductor.
\end{abstract}




\section{Introduction}

Melting of a crystalline solid into a liquid is generally thought of as a disordering process from a state in which the atoms execute thermal motions about static equilibrium sites to one in which all they migrate randomly over the whole space occupied by the material. In a complex system, several different components of this disordering may be distinguished: these can include translational disorder of one sublattice, chemical disorder between two or more sublattices, orientational disorder of molecules or complex ions, or lattice melting in one or two directions in the crystal. When such a system is heated towards the melting point, different dynamical disordering processes set in until complete long-range disorder is achieved in the liquid state. Further distinctions can be made as to whether a particular disordering processes occurs gradually, over an extended temperature range, or suddenly, at a phase transition. If more than one process is associated with a phase transition, these transitions may occur at a single temperature - the melting point (Fig. 1a) - or a sequence of distinct temperatures (Fig. 1b) culminating in the melting point. In either case the entropy of melting may be decomposed into a sum of partial entropies associated with the different disordering processes:

$$
\Delta S=\Delta S_{1}+\Delta S_{2} \ldots+\Delta S_{m}
$$

Along with the atomic structure changes taking place in this way, changes in the transport properties and response functions of the systems, such as electronic conduction thermopower, Hall effect, magnetic susceptibility and ionic conduction, may be expected. These changes may occur either abruptly at a phase transition or gradually over a temperature interval between transitions.

Zintl compounds of the form $A_{m} M_{n}$, where $A$ is an electro-positive (alkali or alkaline earth) metal and $M$ is an electro-negative element, provide a rich ground for investigating this kind of behavior. In these compounds, charge transfer from $A$ to $M$ leads to chemical properties characteristic of elements one or two columns to the right of $M$ in the periodic table, often involving directional bonding and the formation of complex anions. They generally behave as semiconductors or semi metals in both solid and liquid state, and exhibit a rich variety of transport properties, bonding and structure as a function of composition, temperature and pressure. 


\section{Dynamically Disordered Solid Phases}

A typical example of differences in melting behavior is provided by the equiatomic alkali-lead compounds studied by Saboungi and coworkers, which form tetrahedral $\mathrm{Pb}_{4}{ }^{4}$ Zintl ions in both solid and liquid [1]. Fig. 2 shows the enthalpy as a function of temperature for $\mathrm{KPb}$, which exhibits a single transition, and $\mathrm{CsPb}$, which exhibits two transitions about $50 \mathrm{~K}$ apart [2]. To investigate the disordering processes involved, quasielastic neutron scattering (QENS) measurements were carried out at the Institut Laue-Langevin [3], typical results of which are shown in Fig. 3. The spectra in the low-temperature (clearly crystalline) phase (a) could be fitted nicely by a delta function convoluted with the instrumental reduction function, those in the intermediate phase (b) by a combination of delta and Lorentzian functions, and those in high temperature (clearly liquid) phase (c) by a combination of two Lorentzians. These results show immediately that the intermediate phase between the two phase transitions is a solid phase characterized by some kind of dynamic disorder.

The structure factors (intensities as a function of scattering vector $Q$ ) of the two peaks are shown in Fig. 4. Both sets of data are compared with theoretical curves based on a highly simplified model in which $\mathrm{Cs}_{4} \mathrm{~Pb}_{4}$ units, which can be identified in diffraction results [4] from the crystalline phase, exhibit random jump reorientations between the four orientations observed in the crystal. The excellent agreement between this simple model and the data shows that the disordered solid is a rotor phase involving both $\mathrm{Cs}^{+}$and $\mathrm{Pb}_{4}{ }^{4}$ motions.

Similar results for the disordered solid phase in NaSn [5], obtained at ISIS, are shown in Fig. 5. $\mathrm{NaSn}$ is chemically analogous to $\mathrm{CsPb}$, forming $\mathrm{Sn}_{4}{ }^{4} \mathrm{Zintl}$ ions in solid and liquid [6]. The neutron results, however, are somewhat different: in the QENS spectra (Fig. 5a) obtained from measurements made at the high-resolution back-scattering spectrometer IRIS and at the lowerresolution chopper spectrometer HET at two different incident neutron energies, two Lorentzians of different widths can be distinguished at scattering vectors $Q>1 \AA^{-1}$. Furthermore, elastic scattering is not observable expect for the intense Bragg scattering around $1 \AA^{-1}$, despite the large incoherent scattering cross section of $\mathrm{Na}$. These results indicate that the dynamic disorder in $\mathrm{NaSn}$ consists of fast reorientations of the $\mathrm{Sn}_{4}{ }^{4} \mathrm{Zintl}$ ions together with a slower translational migration of the $\mathrm{Na}^{+}$cations [5]. Despite their different time constraints, the two processes must be coupled since only a single phase transition is observed. 


\section{Structure of the Disordered Solids}

Neutron diffraction measurements on the two disordered solid phases just discussed show that the Bragg scattering observed around a wave vector of $1 \AA^{-1}$ is in fact composed of several distinct peaks. This implies crystal structures of relatively low symmetry, surprising since orientational disorder usually proceeds into cubic phases. Diffraction data from the disordered phase of $\mathbf{N a S n}$, taken at IPNS [7], show that this phase is in fact orthorhombic, so that the overall crystal symmetry is actually lowered at the disordering transition.

Rietveld analysis of the diffraction profiles provide a detailed confirmation of the general picture inferred from the QENS data: the $\mathrm{Sn}_{4}{ }^{4} \mathrm{Zintl}$ ions are actually found to occupy two different sites, each with two different orientations, each configuration being occupied one quarter of the time. The $\mathrm{Na}^{+}$cations occupy four different sites, of which two are fixed (presumably helping to maintain the framework of the crystal) and two are mobile, providing pathways for rapid migration parallel to the $c$ axis of the orthorhombic cell. These pathways exhibit pronounced convolutions parallel to the $a$ axis (Fig. 6),

\section{Dynamic Disorder in the Liquids}

Comparison of the QENS data for the disordered solid and liquid phases give insight into the final disordering that takes place on melting [5]. In $\mathrm{CsPb}$, the structure factor of the elastic scattering in the disordered solid phases, shown above in Fig. 4(b), is mimicked by that of the narrower component of the two Lorentzians fitted to the QENS spectra of the liquid (Fig. 7). The energy widths of the two components differ by about a factor 8 . It is reasonable to associate the narrow component with translational diffusions of the centers of mass of the $\mathrm{Pb}_{4}{ }^{4} \mathrm{Zintl}$ ions and the broad component with rotational diffusion of these units and associated motions of the $\mathrm{Cs}^{+}$cations.

In liquid NaSn, the structure factors of the peaks observed in the IRIS and HET measurements follow clearly the behavior observed in the disordered solid phase, shown above in Fig. 5 , the most significant difference being that the Bragg peaks around $1 \AA^{-1}$ are no longer observed [5]. As in the disordered solid phase, it is reasonable to associate the narrow component with the center-of-

mass motions, now including those of the $\mathrm{Sn}_{4}{ }^{4}$ Zintl ions as well as of the $\mathrm{Na}^{+}$cations, and the broad component with the Zintl ion reorientations. 


\section{Vibrational Aspects of the Disorder}

Dynamic disorder can be expected to influence the dynamics on shorter time scales. The characteristics of the vibrational spectra in the three phases of $\mathrm{CsPb}[3]$ are shown in Fig. 8. A regular tetrahedron possesses three normal modes with frequencies in the ratios $1: \sqrt{2}: 2$ (for central forces between the constituent atoms). The $\mathrm{Pb}_{4}{ }^{4}$ Zintl ions can be expected therefore to exhibit a librational mode and three vibrational modes. These can be clearly identified in Fig. 8 (a). As the temperature is increased into the disordered solid (b) and liquid (c) phases, the distinction between orientational and vibrational modes is maintained, although separate vibrational modes can no longer be identified.

\section{Electrical Conductivity}

In a semiconducting compared with a small band gap one would expect a rather subtle interplay between atomic disorder and electronic transport: on the one hand the disorder would tend to increase the scattering of mobile electrons or holes and hence decrease the conductivity, while on the other hand the gap between valence and conduction bands would be expected to decrease, leading to enhanced conductivity. Both effects are in fact observed in the compounds discussed here. A typical behavior is that of NaSn [8] shown in Fig. 9. The conductivity shows a typically activated behavior as temperature is increased in the ordered crystal, drops at the disordering transition and then rises by 2-3 orders of magnitude until the melting point is reached, after which is increases relatively slowly. In $\mathrm{CsPb}$ [8] there is instead a large increase by nearly two orders of magnitude at the disordering transition followed by a slow rise up to the melting point. The general problem of "mixed" conduction, involving interacting fast ions and mobile fermions, has been discussed on a phenomenological basis by Ramasesha [9], who finds a distinction between the "wide-gap" situation characterized by a gradual increase with temperature in population of mobile ions and fermions and the "narrow-gap" situation where both exhibit a jump increase at the disordering transition. In the present context, $\mathrm{NaSn}$ appears to represent the wide-gap case and $\mathrm{CsPb}$ the narrow gap case. A similar distinction is found in other mixed conductor systems, e.g., between $\mathrm{Ag}_{2} \mathrm{Te}$ in the one class and $\mathrm{Ag}_{2} \mathrm{~S}$ and $\mathrm{Ag}_{2} \mathrm{Se}$ in the other [10]. 


\section{Conclusions}

Dynamic disorder in the high-temperature solid phases of the Zintl compounds $\mathrm{CsPb}$ and $\mathrm{NaSn}$ is characterized by fast orientational motions of the polyanions and coupled motions of the cations. In $\mathrm{CsPb}$ these appear to be on a similar time scale, while in $\mathrm{NaSn}$ the orientational motions are more rapid. The cation motions in NaSn appear to be of a translational type in which the $\mathrm{Na}^{+}$ions migrate through the crystal along pathways parallel to the $c$ axis. In both cases melting is characterized by the appearance of slow translational motions of the centers of mass of the polyanions.

The dynamic behaviour of the ions is associated with dramatic increases in electrical conductivity. Here again the detailed behavior shows significant differences, with $\mathrm{CsPb}$ exhibiting the behavior expected of a narrow-gap mixed conductor and NaSn that of a broad-gap gap mixed conductor.

\section{Acknowledgments}

The science reviewed here owes much in spirit to the inspiration of Professor J. E. Enderby, FRS, who was one of the first to appreciate the fundamental connection between structure and transport in disordered condensed matter. This work is supported by the Division of Materials Sciences, Office of Basic.Energy Sciences of the Department of Energy, under Contract No. W-31-109ENG-38. 


\section{References}

[1] Saboungi, M.-L., Blomquist, R., Volin, K. J. and Price , D. L., 1987. J. Chem. Phys. 87, 2278.

[2] Johnson, G. K. and Saboungi, M.-L., 1980. J. Chem. Phys. 86, 6376.

[3] Price, D. L., Saboungi, M.-L. Reijers, H. T. J., Kearley, G. and White, R., 1991. Phys. Rev. Letters 66, 1894; Price, D. L. and Saboungi, M.-L., 1991. Phys. Rèv. B 44, 7289.

[4] Reijers, H. T. J., Saboungi, M.-L., Price, D. L., Richardson, J. W., Volin, K. J. and Van der Lugt, W., 1989. Phys. Rev. B40, 6018.

[5] Saboungi, M.-L. Fortner, J., Howells, W. S. and Price, D. L., 1993. Nature 365, 237; Price, D. L., Saboungi, M.-L. and Howells, W. S., 1995. Phys. Rev. B51, 14923.

[6] Alblas, B. P., van der Lugt, W:, Dijkstra, J., Geertsma, W. and van Dijk, C, 1983. J. Phys. F 13, 2465.

[7] Richardson, J. W., Price., D. L. and Saboungi, M.-L., 1996. Phys. Rev. Letters 76, 1852

[8] Fortner, J.; Saboungi, M.-L. and Enderby, J. E., 1995, Phys. Rev. Letters, 74, 1415.

[9] Ramasesha, S., 1982, J. Solid State Chem. 41, 333.

[10] Junod, P., 1960, Helv. Phys. Acta 32, 567; 601. 


\section{Figure Captions}

1. Schematic illustrations of the melting process in which different disordering processes set in (a) at the same temperature or (b) at a sequence of distinct temperatures.

2. Enthalpy as a function of temperature near the melting points of alkali-lead compounds [2]. The solid lines are a guide to the eye.

3. Quasielastic neutron scattering spectra for the three phases of $\mathrm{CsPb}$ in the vicinity of the phase transitions shown in Fig. 2. Circles: data; solid lines: fitted delta and Lorentzian functions, broadened with experimental energy resolution function [3].

4. Structure factors for (a) Lorentzian and (b) delta-function peaks in the disordered solid phase of $\mathrm{CsPb}(880 \mathrm{~K})$. Circles: data; solid lines: results of the model described in the text [3]. The calculated structure factor in (b) has been broadened with the instrumental $Q$ resolution function.

5. Structure factors for (a) Lorentzian and (b) delta function peaks in the disordered solid phase of NaSn (773K).. Solid circles high resolution data from IRIS; open circles: medium resolution data from HET (neutron energy of $20 \mathrm{meV}$ ); open triangles: low-resolution data from HET (neutron energy of $40 \mathrm{meV}$ ) [5]. The lines are a guide to the eye

6. Fourier contour maps of neutron diffraction in the ac plane of the disordered solid phase of $\mathrm{NaSn}$ at $753 \mathrm{~K}[7]$.

7. Structure factors of the narrow (circles) and broad (triangles) Lorentzian peaks, and total intensities (inverted triangles), for liquid $\mathrm{CsPb}$ at 923K [5]. The lines give a guide to the eye.

8. Generalized vibrational density of states in the three phases of $\mathrm{CsPb}$ [3].

9. Electrical conductivity and thermopower as a function of temperature in $\mathrm{NaSn}$ [8]. 


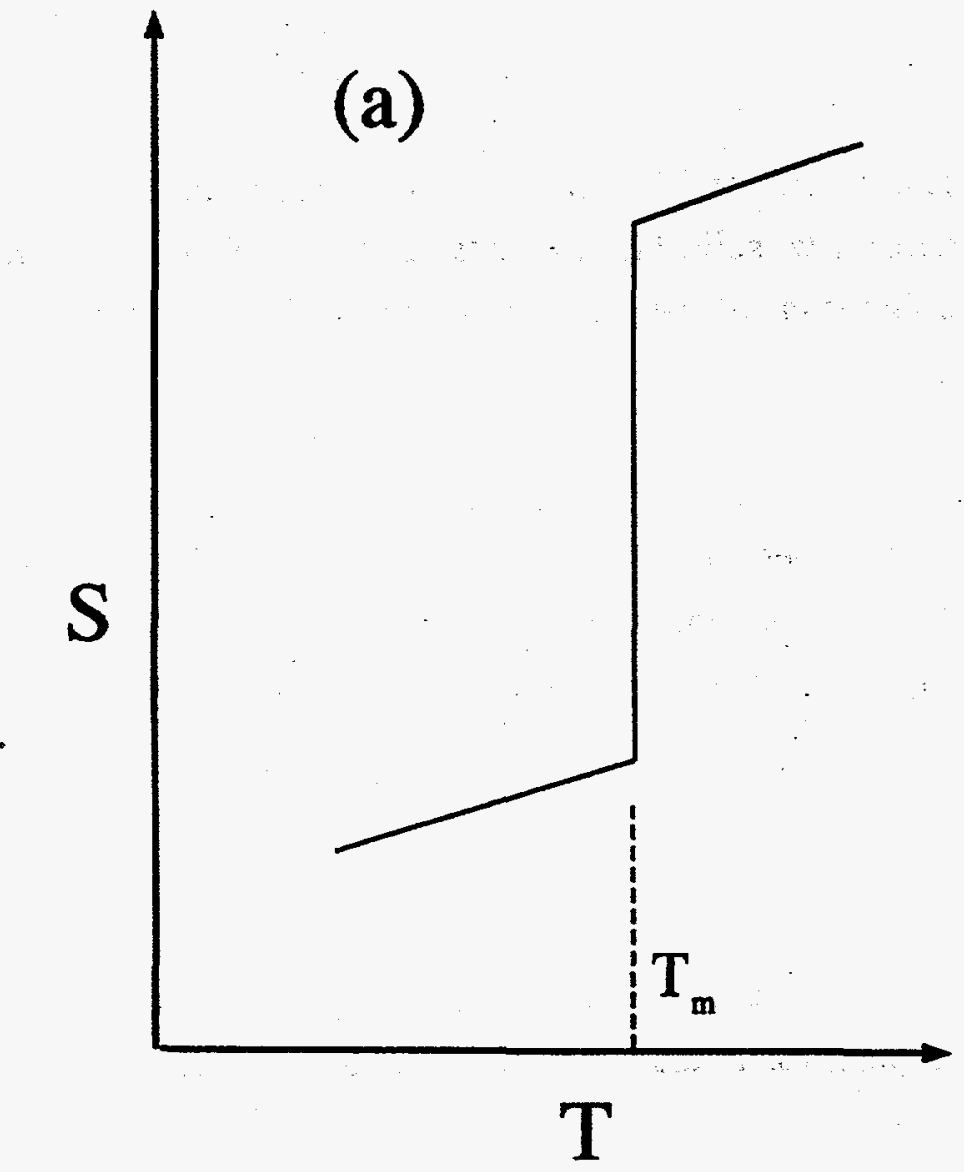

Dree a Satamgi $($ a $)$ 


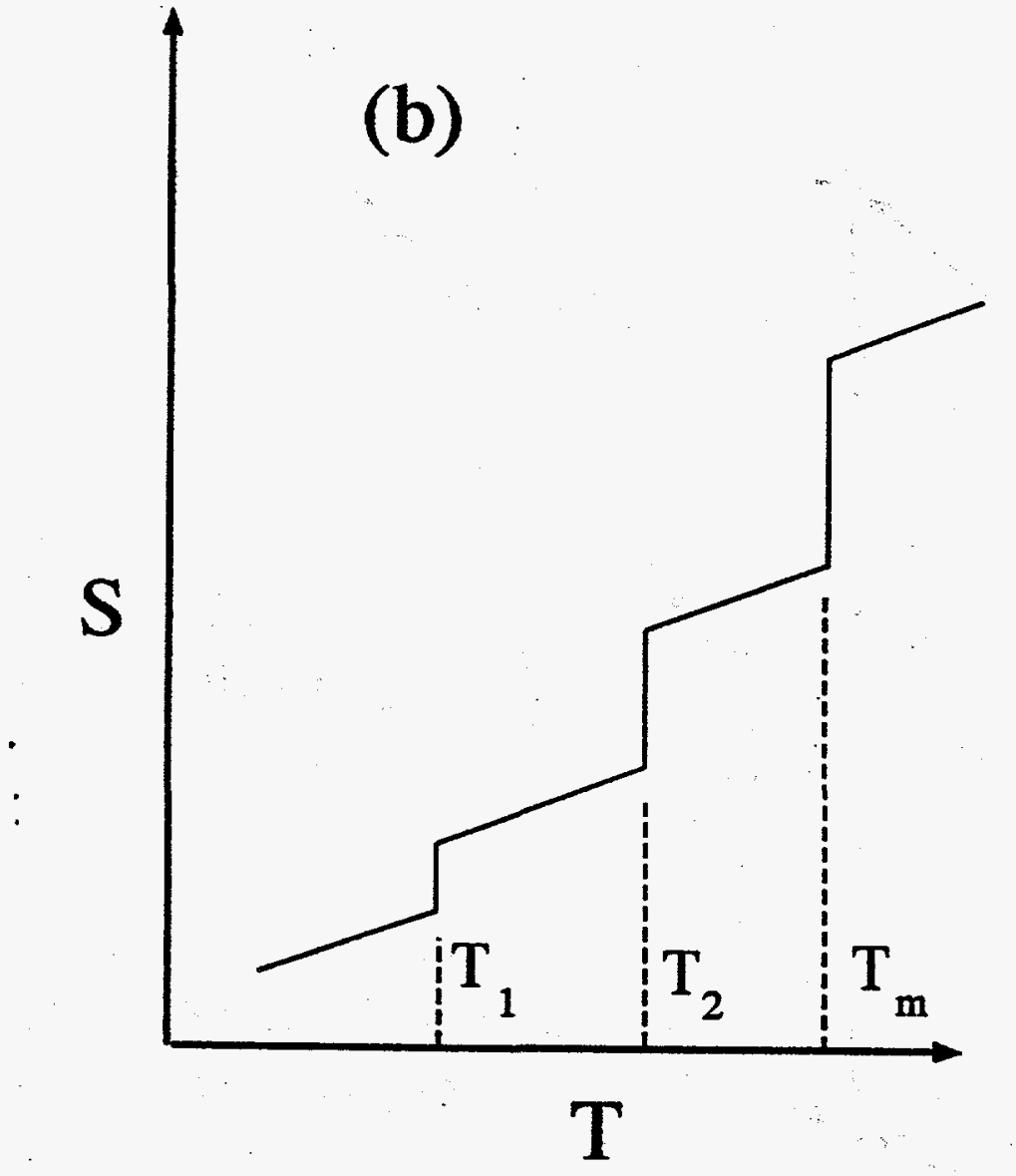

Drie a batrangi $1(t)$ 


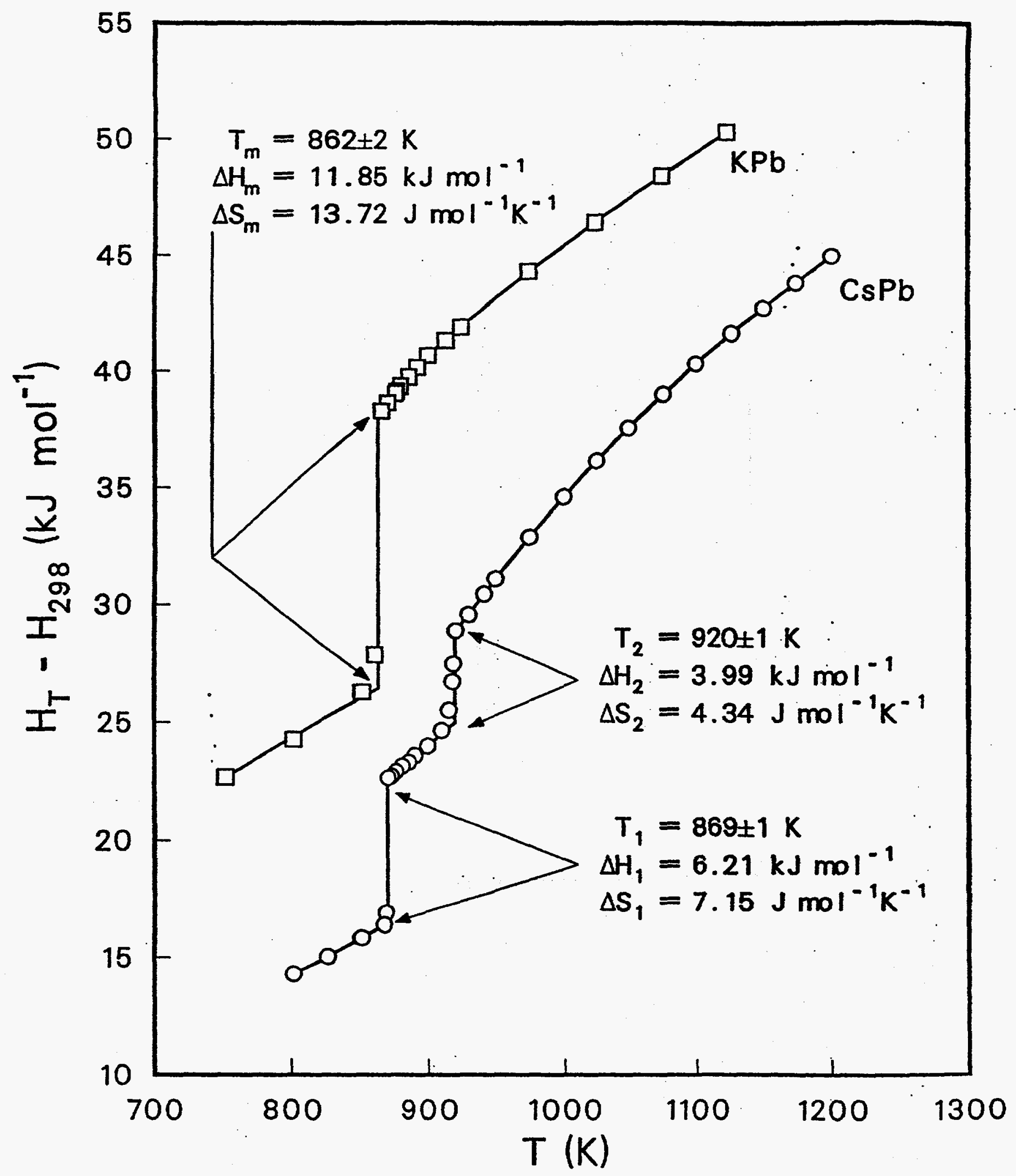

PS Fy 2 

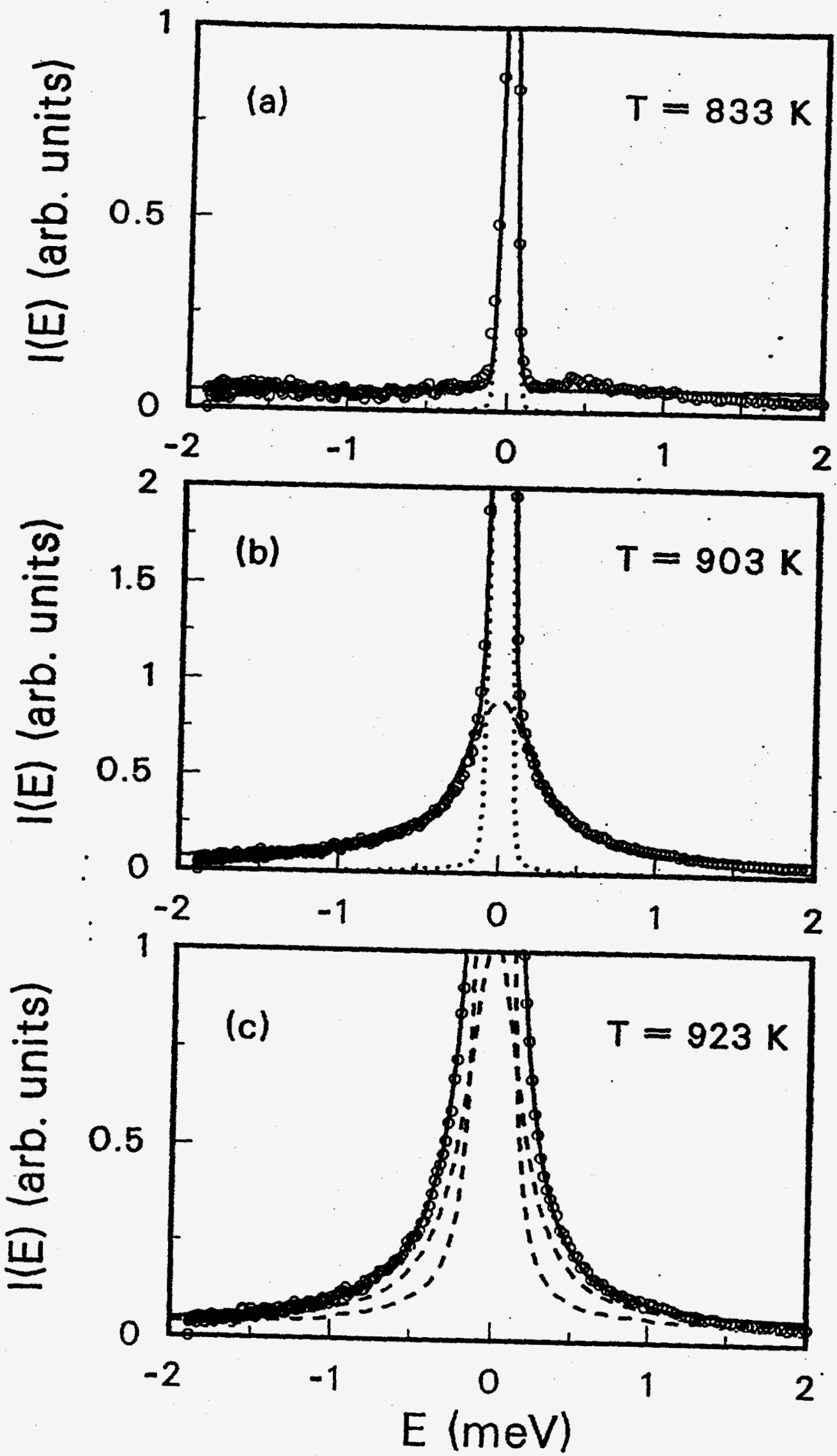

PS Fg. 3 

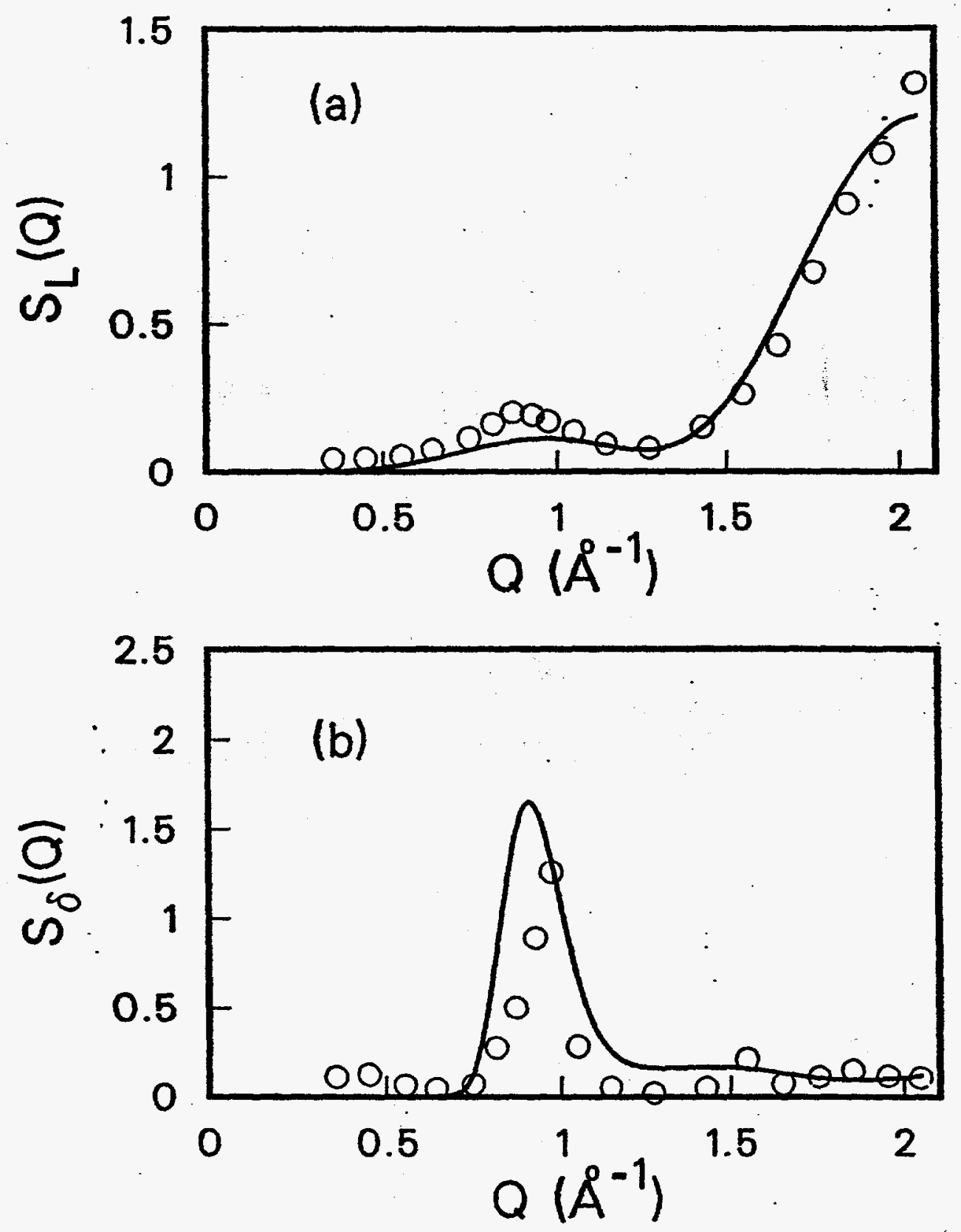

PS $\pi_{9} 4$ 


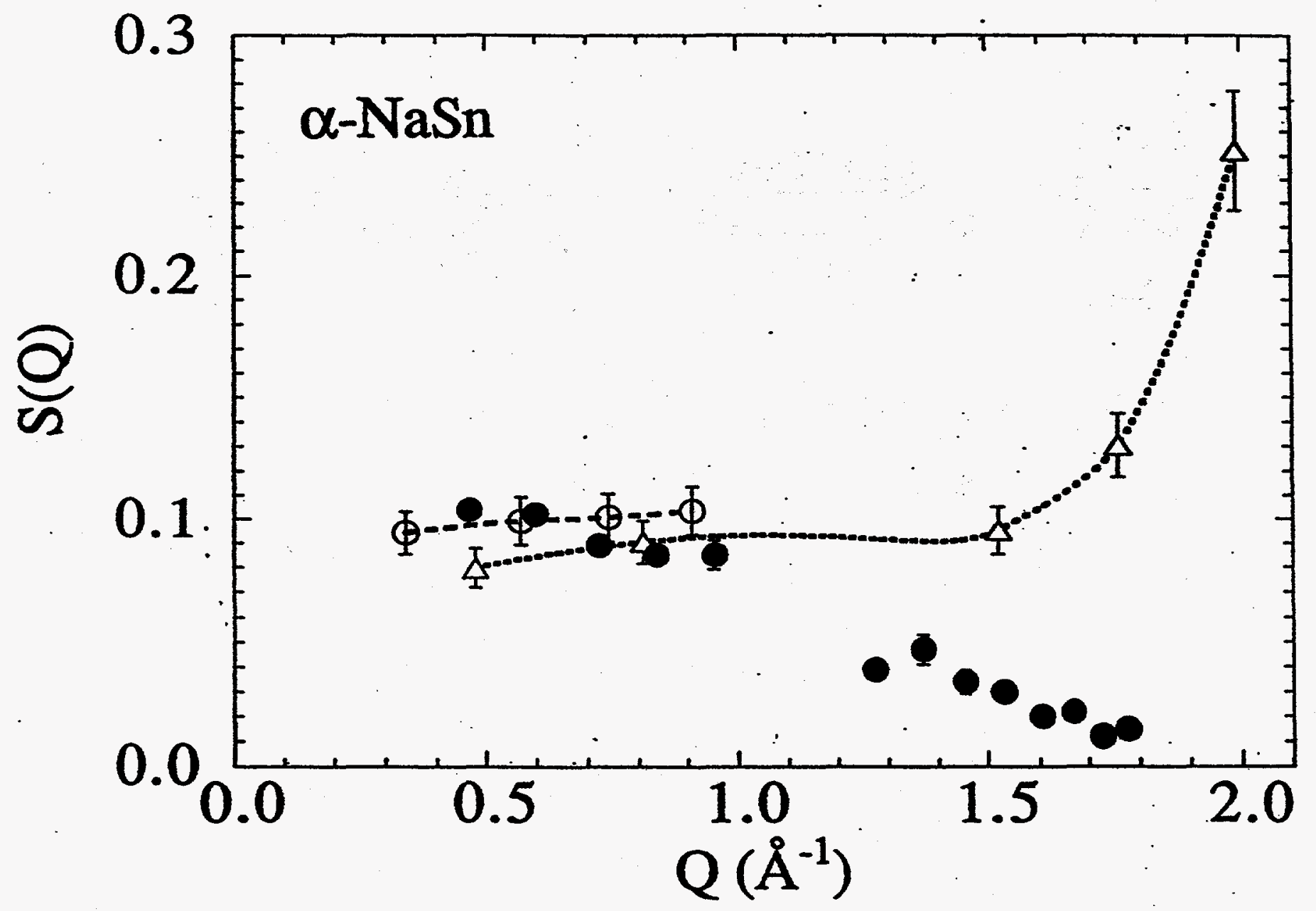

PSFig 5 


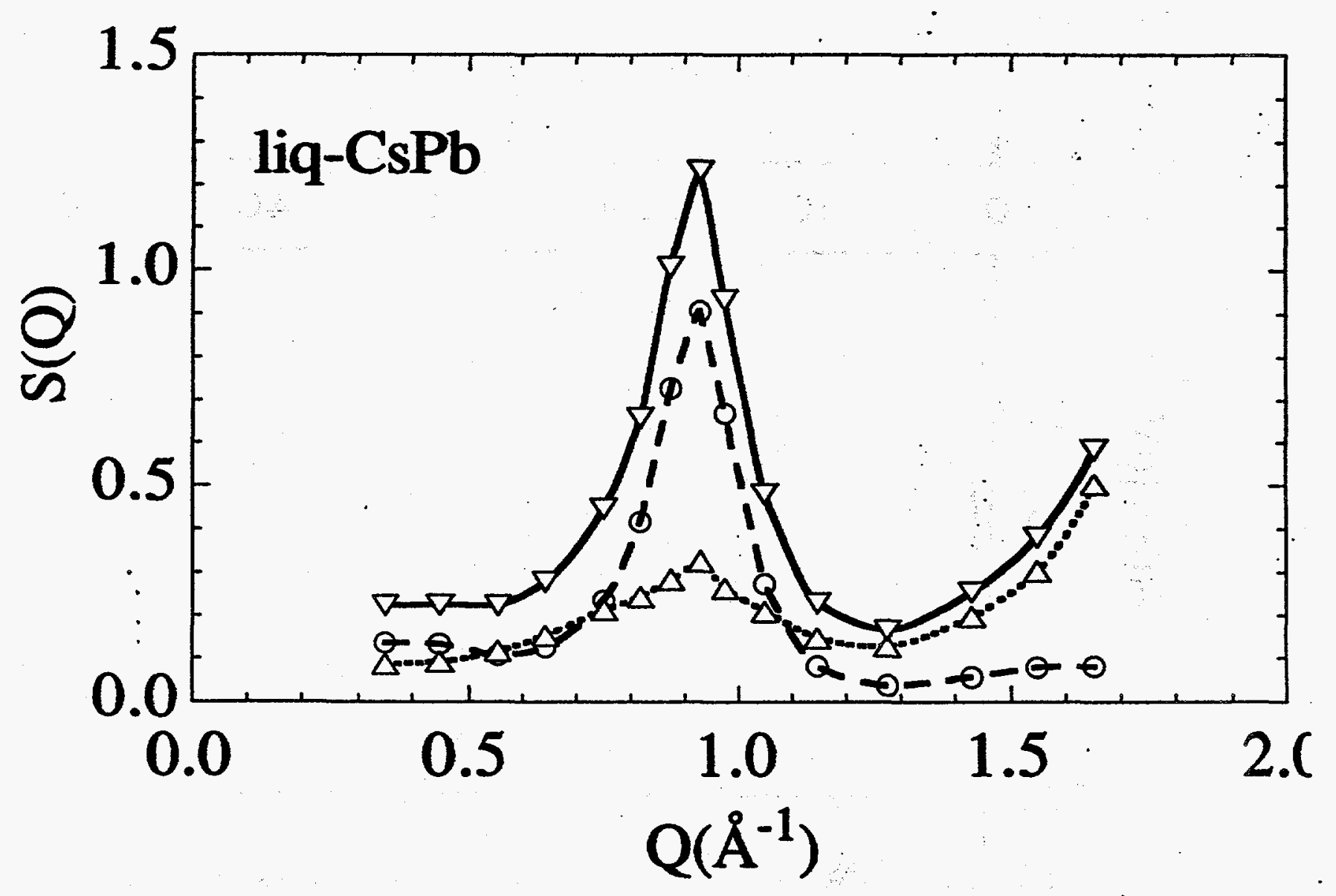


Generalized vibrational density of states
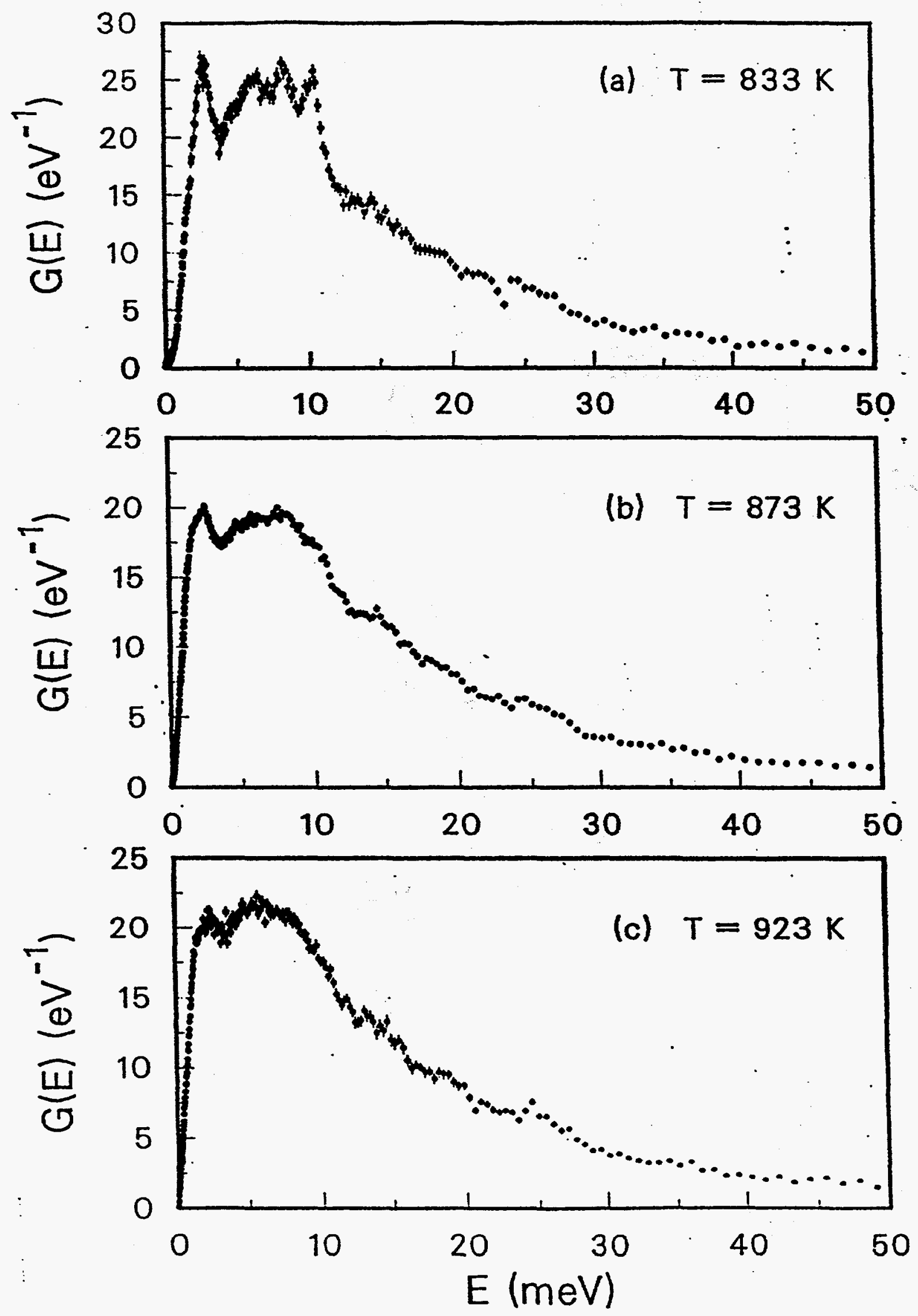

PS Fig. 8 


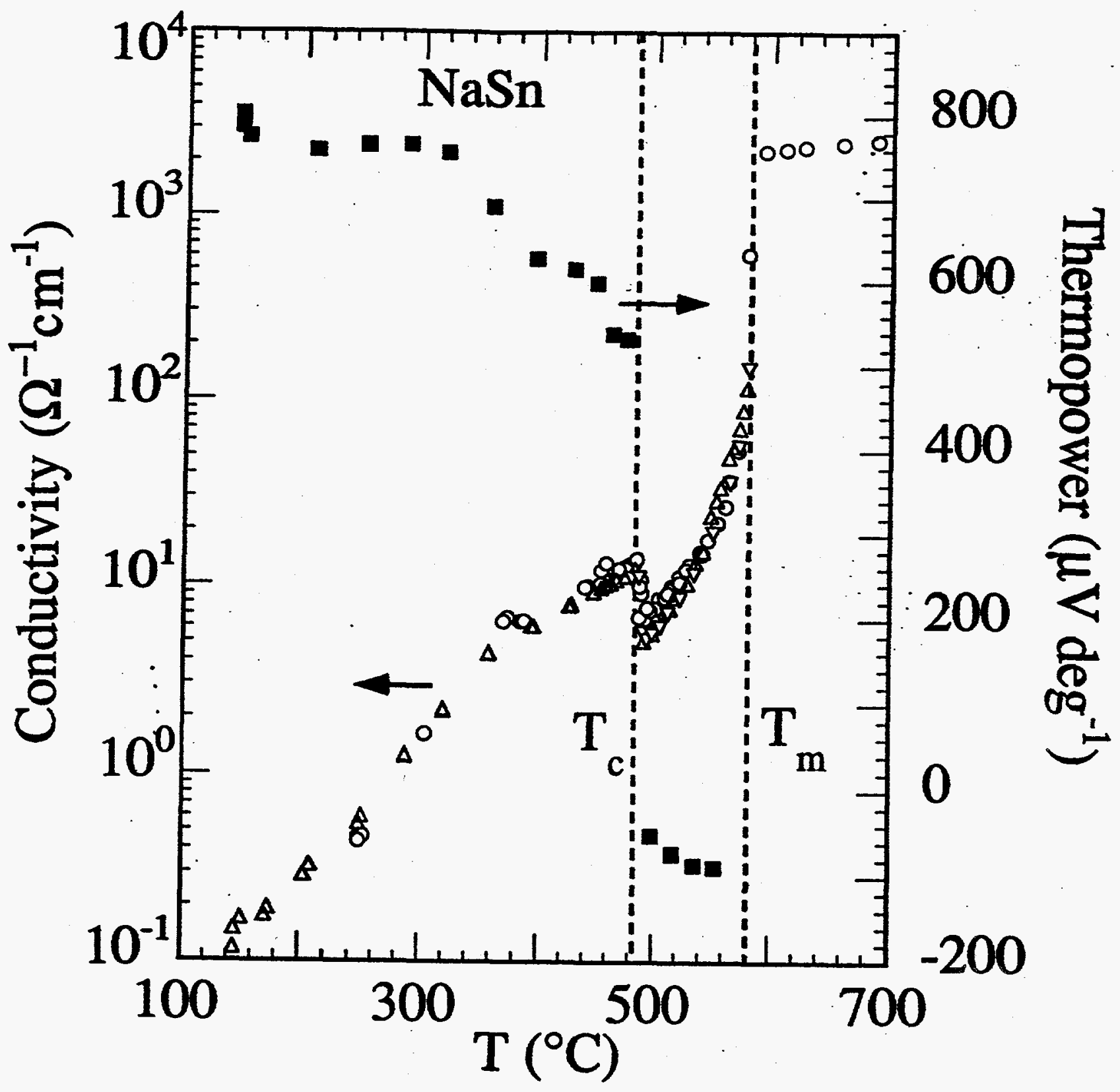

PS Figg 